\title{
Acúmulo de micronutrientes em tango em função do número de hastes cultivadas
}

\author{
Moisés A Muniz; Maria Yumbla-Orbes; José Geraldo Barbosa; Luma G Oliveira
}

UFV, Depto. Fitotecnia, 36570-000 Viçosa-MG; mmuniz76@gmail.com; jgeraldo@ufv.br; maria.yumbla@hotmail.com; luma.oliveira@ ufv.br

\section{RESUMO}

O cultivo de tango no país é recente, sendo importante o conhecimento sobre suas exigências nutricionais para obtenção de melhor manejo da cultura. O objetivo deste trabalho foi avaliar o acúmulo de micronutrientes em plantas de tango submetidas a diferentes sistemas de condução. O experimento foi desenvolvido em delineamento de blocos casualizados, disposto em esquema fatorial $(3 \times 7)$, com três repetições. Cada unidade experimental foi constituída por um vaso contendo três plantas. Os tratamentos foram representados pelo sistema de condução da planta (uma, duas e três hastes por planta) e sete épocas de colheita $[0,15,30,45,60,75$ e 90 dias após plantio (DAP)]. Nas primeiras oito semanas de cultivo, as plantas foram mantidas sob dias longos (DL) com 16 horas de luz, sendo 4 horas por meio de luz artificial para promover o crescimento vegetativo. As plantas foram fertirrigadas com $30 \mathrm{~mL}$ de solução nutritiva nos 30 primeiros dias após transplante e com $50 \mathrm{~mL}$ nos 60 dias posteriores. A fertirrigação foi alternada com a irrigação, de acordo com a necessidade das plantas. Os sistemas de condução não proporcionaram diferença no acúmulo de massa seca das folhas. Não foram observados sintomas de deficiência e toxidez de micronutrientes nas folhas de tango durante o período analisado. $\mathrm{O}$ acúmulo de micronutrientes nas folhas acompanhou o incremento de massa seca durante todo o ciclo, obedecendo à ordem: $\mathrm{Mn}>\mathrm{Fe}>\mathrm{B}>\mathrm{Zn}>\mathrm{Cu}$.

Palavras-chave: Solidago canadensis, ferro, zinco, cobre, manganês, absorção.

\begin{abstract}
Accumulation of micronutrients of goldenrod plants cultivated with different number of stems

The cultivation of goldenrod in Brazil is recent and the knowledge about its nutritional requirements is important to obtain a better crop management. The objective of this study was to evaluate the accumulation of micronutrients in plants of goldenrod under different management systems. The experiment was carried out in a randomized block design, arranged in a factorial scheme $(3 \times 7)$ with three replications. Each experimental unit consisted of one pot containing three plants. The treatments were represented by the conduction system of the plant (one, two and three stems per plant) and seven harvest times $[0,15,30,45,60,75$ and 90 days after planting (DAP)]. During the first eight weeks of cultivation the plants were maintained under long day (LD) with 16 hours of light (4 additional hours by means of artificial light to promote vegetative growth). The plants were fertigated with $30 \mathrm{~mL}$ of nutrient solution in the first 30 days after planting and $50 \mathrm{~mL} 60$ days later. The drip irrigation was alternated with irrigation according to the need of the plants. The conduction system did not provide difference in dry mass of leaves. There were no symptoms of micronutrient deficiency and toxicity in the leaves of goldenrod during the period analyzed. The accumulation of micronutrients in the leaves followed the increase of dry mass throughout plant growth, following the order: $\mathrm{Mn}>\mathrm{Fe}>\mathrm{B}>\mathrm{Zn}>\mathrm{Cu}$.
\end{abstract}

Keywords: Solidago canadensis, iron, zinc, copper, manganese, absorption.

(Recebido para publicação em 5 de setembro de 2012; aceito em 24 de abril de 2013) (Received on September 5, 2012; accepted on April 24, 2013)

$\mathrm{O}$ tango (Solidago canadensis), originário da América do Norte, da família Asteraceae, é uma planta ornamental herbácea, rizomatosa, perene, pouco ramificada, que alcança de 0,8 a $1,2 \mathrm{~m}$ de altura. Apresenta inflorescências verde-amarelas terminais grandes, eretas, ramificadas, com numerosos capítulos pequenos. Devido às suas belas inflorescências, é comercializada como flor de corte para buquês ou arranjos florais (Lorenzi \& Souza, 2001).

No Brasil, o cultivo comercial do tango é recente com muitos problemas quanto aos tratos culturais necessários para aumentar a eficiência produtiva, particularmente a nutrição, uma vez que tanto os fatores nutricionais quanto o sistema de condução das plantas estão diretamente relacionados com a produtividade.

Prática comum entre os produtores de tango é a realização da poda, resultando na superação da dominância apical propiciando a emissão de duas a quatro hastes por planta, conforme observado por Cuquel et al. (1999). Assim para o adequado acúmulo de biomassa e produção de hastes comerciais, características indispensáveis para uma rentável produtividade agrícola, a exigência por micronutrientes pela planta devem ser suprida (Paulus et al. 2007).

Os micronutrientes são exigidos pelas plantas em quantidades muito baixas para adequado crescimento e desenvolvimento das plantas, mas apresentam a mesma importância dos macronutrientes para a nutrição. Teores inadequados limitam o crescimento e desenvolvimento, como também reduzem a eficiência de uso dos macronutrientes. Além disso, os micronutrientes, particularmente o $\mathrm{Cu}, \mathrm{Mn}, \mathrm{Zn}, \mathrm{B}$ estão particularmente envolvidos na fase reprodutiva do 
crescimento, e, conseqüentemente, na determinação da produtividade e da qualidade da planta, enquanto o ferro está envolvido na síntese de clorofila e na cadeia de transporte de elétrons de ambos fotossistemas (Kirkby \& Römheld, 2007). Informações técnicas a respeito de como a poda interfere no crescimento e desenvolvimento da planta e como esta interfere na demanda por nutrientes, principalmente em relação aos micronutrientes, ainda não foram pesquisadas para a cultura do tango. Assim, o objetivo deste trabalho foi determinar o acúmulo de massa seca e de micronutrientes em plantas de tango conduzidas com uma, duas e três hastes.

\section{MATERIAL E MÉTODOS}

O experimento foi desenvolvido em condição de casa de vegetação da UFV, em Viçosa-MG (2045'S, 42 $5^{\circ}$ 'O, altitude de $651 \mathrm{~m}$ ). As mudas de tango foram obtidas de estacas herbáceas de $10 \mathrm{~cm}$, retiradas de plantas matrizes cultivadas no setor de floricultura. Após o enraizamento as mudas foram transplantadas para vasos com capacidade de 1,5 L, contendo areia lavada como substrato.

Utilizou-se o delineamento em blocos casualizados, disposto em esquema fatorial $(3 \times 7)$, com três repetições. Os tratamentos foram constituídos por três sistemas de condução da planta (uma, duas e três hastes por planta), e sete épocas de colheita $[0,15,30,45,60,75$ e 90 dias após o plantio (DAP)]. Cada unidade experimental foi constituída por um vaso contendo três plantas.

Aos cinco dias após o transplantio, efetuou-se a poda, que consistiu na eliminação de um centímetro do ápice da planta. Nas oito semanas de cultivo, as plantas foram mantidas sob dias longos (DL), para promover o crescimento vegetativo, com 16 horas de luz, sendo quatro horas por meio de luz artificial. Para isso foi montado sistema de iluminação com lâmpadas de 100 watts, espaçadas $2 \mathrm{~m}$ entre si e instaladas a $1 \mathrm{~m}$ de altura das plantas, controladas por temporizador, ligando as lâmpadas das 22 às $2 \mathrm{~h}$.

As plantas foram fertirrigadas com $30 \mathrm{~mL}$ nos 30 primeiros dias após o plantio e com $50 \mathrm{~mL}$ nos 60 dias poste- riores com solução nutritiva, utilizada por Muniz et al. (2009), contendo, 10,8; 3,$6 ; 1,95 ; 8,0 ; 1,50 ; 1,0$ e $0,5 \mathrm{mmol} / \mathrm{L} \mathrm{de}$

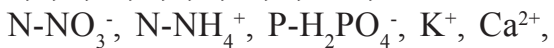
$\mathrm{Mg}^{2+}, \mathrm{S}_{-} \mathrm{SO}_{4}{ }^{2-}$ e 30,$0 ; 5,0 ; 50,0 ; 40,0$; 0,2 ; e $0,1 \mu \mathrm{mol} / \mathrm{L}$ de $\mathrm{B}, \mathrm{Cu}, \mathrm{Fe}, \mathrm{Mn}$, $\mathrm{Zn}$, Mo respectivamente. A fertirrigação foi alternada com a irrigação, de acordo com a necessidade.

Em cada colheita as plantas eram imediatamente separadas em folhas, caule, raiz e inflorescência. Depois de colhido, o material foi levado ao laboratório, lavado em água destilada e colocado em sacos de papel para secagem em estufa de circulação forçada de ar, a $70-75^{\circ} \mathrm{C}$, por 72 horas. Em seguida, todas as folhas foram pesadas, moídas, em moinho tipo Willey, utilizando-se peneira de 40 mesh, e digeridas com mistura nitríco-perclórica. No extrato obtido, foi efetuada a determinação dos teores totais de $\mathrm{Fe}, \mathrm{Zn}, \mathrm{Cu}$ e $\mathrm{Mn}$ por espectrofotometria de absorção atômica. Para determinação do boro, amostras de $0,25 \mathrm{~g}$ foram calcinadas por $3 \mathrm{~h}$, a $550^{\circ} \mathrm{C}$, em cadinhos de porcelana. Em seguida, o resíduo da calcinação foi dissolvido em $10 \mathrm{~mL}$ de acido clorídrico $0,1 \mathrm{~mol}$ $\mathrm{L}^{-1}$ e passado por filtro com poro de 0,45 $\mu \mathrm{m}$. No extrato obtido, foi determinado o teor de boro por espectrofotometria de emissão ótica, com plasma acoplado por indução (ICP-OES).

A determinação do acúmulo de nutrientes (microgramas por planta) foi efetuada multiplicando-se a massa seca de folha (mg) pelo teor do micronutriente $\left(\mathrm{mg} \mathrm{kg}^{-1}\right)$.

$$
\text { Acúmulo }\left({ }^{\mu g} / \text { planta }\right)=\frac{M S \times C N}{1000}
$$

Os resultados obtidos foram submetidos à análise de variância e, em função do nível de significância no teste F para os tratamentos, procedeu-se ao estudo de regressão. As médias foram comparadas, utilizando-se o teste de Newman Keuls, adotando-se o nível de $5 \%$ de probabilidade para os tratamentos qualitativos (condução das plantas). Para os tratamentos quantitativos (coletas durante o ciclo da cultura) foram ajustados modelos de regressão, sendo selecionadas dentro da equação de regressão, aquelas de efeito significativo, pelo teste $\mathrm{F}$, a 1 e $5 \%$ de probabilidade,

Tabela 1. Massa fresca e comprimento de hastes em plantas conduzidas com uma, duas e três haste aos 90 DAP (fresh weight and length of branches in plants conducted with one, two and three branches on 90 DAP). Viçosa, UFV, 2008.

\begin{tabular}{lcc}
\hline No hastes & Massa fresca $(\mathbf{g})$ & Comprimento de hastes $(\mathbf{c m})$ \\
\hline 1 & $87,19 \mathrm{a}$ & $108,33 \mathrm{a}$ \\
2 & $82,01 \mathrm{a}$ & $92,17 \mathrm{a}$ \\
3 & $73,52 \mathrm{a}$ & $71,44 \mathrm{~b}$ \\
\hline DMS & 18,11 & 35,54 \\
\hline
\end{tabular}

Médias seguidas pela mesma letra na coluna não diferem entre si pelo teste de Newman Keuls a $5 \%$ de probabilidade (means followed by same letter in column do not differ by Newman Keuls test at $5 \%$ probability).

Tabela 2. Massa seca de folhas (g) de plantas conduzidas com uma, duas e três hastes em diferentes épocas de avaliação após o plantio (leaf dry mass $(\mathrm{g})$ of plants conducted with one, two and three branches at different evaluation dates after planting). Viçosa, UFV, 2008.

\begin{tabular}{lccccccc}
\hline \multirow{2}{*}{ No hastes } & \multicolumn{7}{c}{ Época de avaliação (dias) } \\
\cline { 2 - 8 } & $\mathbf{0}$ & $\mathbf{1 5}$ & $\mathbf{3 0}$ & $\mathbf{4 5}$ & $\mathbf{6 0}$ & $\mathbf{7 5}$ & $\mathbf{9 0}$ \\
\hline 1 & $0,30 \mathrm{a}$ & $0,21 \mathrm{a}$ & $0,77 \mathrm{a}$ & $2,07 \mathrm{a}$ & $2,77 \mathrm{a}$ & $3,48 \mathrm{a}$ & $4,42 \mathrm{a}$ \\
2 & $0,22 \mathrm{a}$ & $0,19 \mathrm{a}$ & $0,42 \mathrm{a}$ & $1,71 \mathrm{a}$ & $3,02 \mathrm{a}$ & $2,93 \mathrm{a}$ & $3,49 \mathrm{a}$ \\
3 & $0,28 \mathrm{a}$ & $0,17 \mathrm{a}$ & $0,63 \mathrm{a}$ & $1,91 \mathrm{a}$ & $3,80 \mathrm{a}$ & $3,01 \mathrm{a}$ & $3,87 \mathrm{a}$ \\
\hline DMS & 0,10 & 0,07 & 0,37 & 0,38 & 1,05 & 0,51 & 0,94 \\
\hline
\end{tabular}

Médias seguidas pela mesma letra na coluna não diferem entre si pelo teste de Newman Keuls a $5 \%$ de probabilidade (means followed by same letter in column do not differ by Newman Keuls test at $5 \%$ probability). 
de significado biológico e de maior soma de quadrado, ou seja, maior $\mathrm{R}^{2}$. A análise estatística foi realizada com o auxílio do programa SAEG.

\section{RESULTADOS E DISCUSSÃO}

A quantidade de massa fresca produzida pelo tango nos três sistemas de condução foi superior que o indicado para a classificação super (35 g) pelas normas de qualidade do Veiling (2011), que é o maior entreposto brasileiro de comercialização da cultura. Entretanto, não houve diferença entre os sistemas de condução com uma, duas ou três hastes colhidas aos 90 dias quanto à massa seca de haste floral (Tabela 1), ao contrário do esperado. Segundo Wachowiz \& Carvalho (2002), em algumas espécies, o aumento do número de hastes cultivadas promove competição entre as plantas e, consequentemente, ocorre diminuição de tamanho das flores, comprimento e diâmetro das hastes, além de redução da massa fresca das hastes florais.

Os sistemas de condução com uma e duas hastes proporcionaram comprimento de haste superior ao sistema com três hastes aos 90DAP (Tabela 1). Estes resultados são semelhantes aos observado por Cuquel et al. (1999) que encontraram altura de $100,7 \mathrm{~cm}$ em plantas de tango cultivadas com uma haste e sem utilização de poda. O maior comprimento das hastes em plantas com uma ou duas hastes pode ser devido à menor competição por fotoassimilados, água e nutrientes dentro da planta, uma vez que o crescimento vertical e único favorece a elongação dos entre-nós (Wachowiz \& Carvalho, 2002).

Segundo a classificação do Veiling (2011), em que o comprimento das hastes varia de 50 a $90 \mathrm{~cm}$, os sistemas com uma e duas hastes foram enquadrados em melhores categorias em relação àquele conduzido com três hastes. Esses sistemas foram rentáveis ao produtor, pois o comprimento das hastes e a massa fresca definem o valor comercial do tango. Entretanto, como não ocorreu diferença na produção de massa fresca, mesmo com menor comprimento de

Tabela 3. Acúmulo de ferro, cobre, manganês, zinco e boro em folhas de plantas de tango conduzidas com uma, duas e três hastes em diferentes épocas de avaliação após plantio (accumulation of iron, copper, manganese, zinc and boron in plant leaves of goldenrod conducted with one, two and three branches at different times of evaluation after planting). Viçosa, UFV, 2008.

\begin{tabular}{|c|c|c|c|c|c|c|c|}
\hline \multirow{3}{*}{$\begin{array}{l}N^{0} \text { de } \\
\text { hastes }\end{array}$} & \multicolumn{7}{|c|}{$\mathrm{Fe}(\mu \mathrm{g} /$ planta $)$} \\
\hline & \multicolumn{7}{|c|}{ Época de avaliação (DAP) } \\
\hline & $\mathbf{0}$ & 15 & 30 & 45 & 60 & 75 & 90 \\
\hline 1 & $71,27 \mathrm{a}$ & 75,16 a & $177,35 \mathrm{a}$ & $248,40 \mathrm{~b}$ & $505,31 \mathrm{a}$ & $988,97 \mathrm{a}$ & $1450,29 \mathrm{a}$ \\
\hline 2 & $52,82 \mathrm{a}$ & $55,33 \mathrm{a}$ & $106,20 \mathrm{a}$ & $571,71 \mathrm{ab}$ & 654,82 a & 945,36 a & $1178,01 \mathrm{a}$ \\
\hline 3 & $65,51 \mathrm{a}$ & $53,54 \mathrm{a}$ & $151,23 \mathrm{a}$ & $835,84 \mathrm{a}$ & $545,96 \mathrm{a}$ & $1030,83 \mathrm{a}$ & $1303,14 \mathrm{a}$ \\
\hline \multirow[t]{2}{*}{ DMS } & 19,45 & 22,62 & 72,15 & 263,13 & 150,51 & 95,47 & 148,15 \\
\hline & \multicolumn{7}{|c|}{$\mathrm{Cu}(\mu \mathrm{g} /$ planta $)$} \\
\hline 1 & $16,12 \mathrm{a}$ & $10,81 \mathrm{a}$ & 46,81 a & 47,19 a & $22,15 \mathrm{a}$ & $60,35 \mathrm{a}$ & $75,39 \mathrm{a}$ \\
\hline 2 & $11,95 \mathrm{a}$ & $9,99 \mathrm{a}$ & $20,22 \mathrm{~b}$ & $28,49 \mathrm{a}$ & 23,85 a & $50,37 \mathrm{a}$ & $47,36 \mathrm{~b}$ \\
\hline 3 & $14,82 \mathrm{a}$ & $8,09 \mathrm{a}$ & $21,50 \mathrm{~b}$ & $32,50 \mathrm{a}$ & $34,16 \mathrm{a}$ & $61,99 \mathrm{a}$ & $68,17 \mathrm{ab}$ \\
\hline \multirow[t]{2}{*}{ DMS } & 5,17 & 3,62 & 25,59 & 19,77 & 13,01 & 12,62 & 6,77 \\
\hline & \multicolumn{7}{|c|}{ Mn ( $\mu$ g/planta) } \\
\hline 1 & $18,38 \mathrm{a}$ & $37,12 \mathrm{a}$ & $181,19 \mathrm{a}$ & $687,68 \mathrm{a}$ & $1014,43 \mathrm{a}$ & $2230,71 \mathrm{a}$ & $2929,79 \mathrm{a}$ \\
\hline 2 & $13,62 \mathrm{a}$ & 34,98 a & 131,49 a & $376,41 \mathrm{a}$ & 959,63 a & $1308,59 \mathrm{~b}$ & $2461,37 \mathrm{a}$ \\
\hline 3 & $16,90 \mathrm{a}$ & $35,98 \mathrm{a}$ & $201,18 \mathrm{a}$ & $553,82 \mathrm{a}$ & $1441,86 \mathrm{a}$ & $1533,52 \mathrm{~b}$ & $2307,04 \mathrm{a}$ \\
\hline \multirow[t]{2}{*}{ DMS } & 5,76 & 3,14 & 70,69 & 312,27 & 428,43 & 696,19 & 623,75 \\
\hline & \multicolumn{7}{|c|}{ Zn ( $\mu$ g/planta) } \\
\hline 1 & $15,87 \mathrm{a}$ & $8,12 \mathrm{a}$ & $18,30 \mathrm{a}$ & $38,89 \mathrm{a}$ & $40,29 \mathrm{a}$ & $79,79 \mathrm{a}$ & $137,02 \mathrm{a}$ \\
\hline 2 & $11,76 \mathrm{a}$ & $6,74 \mathrm{a}$ & $14,28 \mathrm{a}$ & 37,77 a & $27,57 \mathrm{a}$ & $49,45 \mathrm{a}$ & $88,08 \mathrm{~b}$ \\
\hline 3 & $14,59 \mathrm{a}$ & $6,37 \mathrm{a}$ & $24,08 \mathrm{a}$ & $45,31 \mathrm{a}$ & $38,71 \mathrm{a}$ & $56,33 \mathrm{a}$ & $97,31 \mathrm{~b}$ \\
\hline \multirow[t]{2}{*}{ DMS } & 5,11 & 2,75 & 10,98 & 7,42 & 13,72 & 31,34 & 49,94 \\
\hline & \multicolumn{7}{|c|}{ B ( $\mu \mathrm{g} /$ planta $)$} \\
\hline 1 & $11,39 \mathrm{a}$ & $13,57 \mathrm{a}$ & $65,23 \mathrm{a}$ & $122,27 \mathrm{a}$ & $179,76 \mathrm{a}$ & $225,57 \mathrm{a}$ & $164,94 \mathrm{a}$ \\
\hline 2 & $8,44 \mathrm{a}$ & $12,06 \mathrm{a}$ & $55,75 \mathrm{a}$ & $104,23 \mathrm{a}$ & $131,91 \mathrm{a}$ & 196,14 a & $122,40 \mathrm{a}$ \\
\hline 3 & $10,47 \mathrm{a}$ & $10,57 \mathrm{a}$ & $109,86 \mathrm{a}$ & $172,17 \mathrm{a}$ & $190,51 \mathrm{a}$ & $158,32 \mathrm{a}$ & 156,23 a \\
\hline DMS & 3,95 & 4,00 & 55,11 & 68,94 & 59,60 & 68,25 & 43,54 \\
\hline
\end{tabular}

Médias seguidas pela mesma letra na coluna não diferem entre si pelo teste de Newman Keuls a 5\% de probabilidade (means followed by same letter in column do not differ by Newman Keuls test at $5 \%$ probability). 


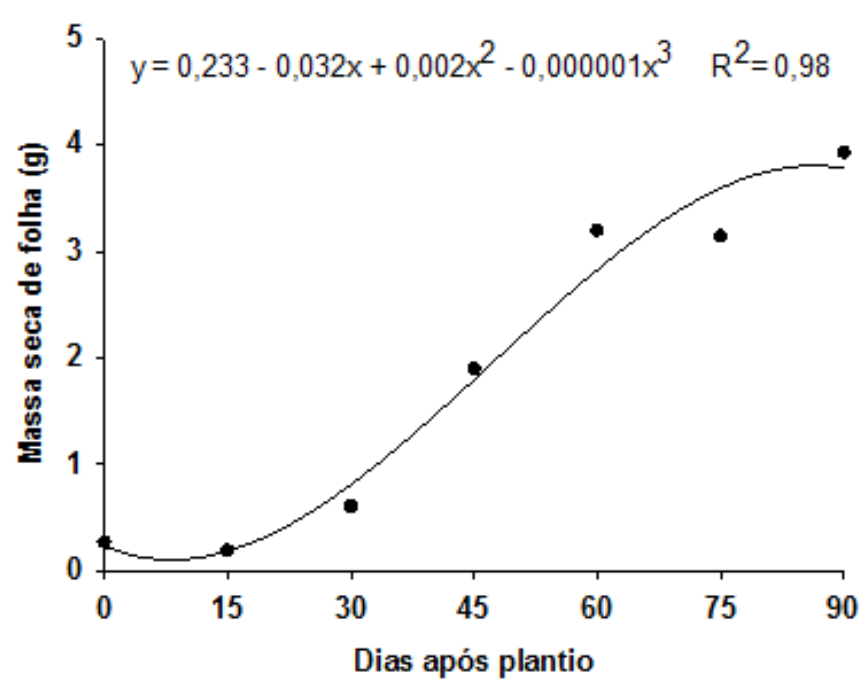

Figura 1. Acúmulo de massa seca de folha em plantas de tango (y), em g/planta, em função dos dias após plantio, na média do número de hastes conduzidas (accumulation of dry mass on leaves of goldenrod plants (y), in g/plant, depending on days after planting, based on the mean among treatments with different numbers of conducted branches). Viçosa, UFV, 2008.

hastes, o sistema de condução com três hastes poder ter sido o mais rentável, uma vez que a produção é diretamente proporcional ao número de hastes por planta.

Não houve diferença para massa seca de folha das plantas conduzidas com uma, duas e três hastes em todas as épocas de avaliação (Tabela 2). A tendência de diminuição da massa seca entre o plantio até os 15 dias após plantio ocorreu devido à poda de condução realizada aos 5 dias após plantio (Tabela 2). Os valores médios da massa seca das folhas ao longo das épocas avaliadas se ajustaram ao modelo de regressão cúbico (Figura 1), sendo o valor máximo estimado de 3,8 g, aos 86 DAP. O aumento de volume na planta ao longo do tempo é conseqüência do crescimento vegetativo, uma vez que o crescimento é definido como o aumento irreversível de massa. O maior componente do crescimento vegetal é a expansão celular e, durante este processo, as células aumentam várias vezes em volume (Taiz $\&$ Zeiger, 2008).

Quanto aos micronutrientes, não foram constatadas diferenças entre os tratamentos para o acúmulo de ferro nas folhas das plantas nas diferentes épocas avaliadas, exceto aos 45 dias em que o sistema de condução com três hastes proporcionou acúmulo de ferro superior ao sistema de condução com uma haste (Tabela 3). De forma semelhante ao ocorrido no presente experimento para o acúmulo de ferro ao longo das épocas (Figura 2), Pedrosa (1998) observou que o acúmulo de ferro em gipsofila (Gypsophila paniculata) foi crescente em todos os órgãos da planta, sendo que a raiz foi o órgão que mais acumulou este micronutriente durante o ciclo, seguido da folha, da inflorescência e do caule. $\mathrm{O}$ acúmulo de ferro nas folhas foi crescente e acompanhou o incremento de biomassa das folhas. Aos 90 dias após plantio foram observados os acúmulos máximos, dentro do intervalo analisado, de 1471,$28 ; 1162,41$ e $1280,39 \mu \mathrm{g} /$ planta em plantas conduzidas com uma, duas e três hastes, respectivamente. Já para Aster ericoides, Camargo et al. (2005) encontraram acúmulo de $1714 \mu \mathrm{g}$ de Fe/ planta aos 90 dias após o plantio.

Não foram observadas diferenças significativas para acúmulo de cobre nas folhas das plantas conduzidas com uma, duas e três hastes aos $0,15,45,60$ e 75 DAP. Por outro lado, aos 30 e 90 DAP, o acúmulo máximo foi observado em plantas conduzidas com uma haste, como consequência do maior acúmulo de massa seca propiciado por este sistema de condução (Tabela 3). Durante o período avaliado houve aumento do acúmulo de cobre, observando-se valores mínimos nas folhas na primeira colheita com 11,10;11,47 e 13,29 $\mu \mathrm{g} /$ planta e valores máximos aos 90 dias com 69,$57 ; 49,45$ e $76,04 \mu \mathrm{g} /$ planta para as plantas conduzidas com uma, duas e três hastes, respectivamente. Esses valores estão próximos dos encontrados por Camargo et al (2005), que estudando a absorção de micronutrientes em aster, observaram acúmulo de cobre de $77,5 \mu \mathrm{g} /$ planta aos 75 dias após o transplantio. Já Ferreira (2009), estudando crisântemo, encontrou acúmulo de 218 $\mu \mathrm{g}$ de cobre por planta.

Quanto ao acúmulo de manganês nas folhas, não foram observadas diferenças estatísticas entre os sistemas de condução nas diferentes épocas avaliadas, exceto aos $75 \mathrm{DAP}$, quando o sistema de condução com uma haste proporcionou acúmulo superior aos demais. (Tabela 3). O acúmulo de manganês nas folhas das plantas conduzidas com uma, duas e três hastes foi ajustado ao modelo de regressão quadrático (Figura 2), sendo o máximo de 3009,$53 ; 2355,72$ e $2326,38 \mu \mathrm{g}$ de $\mathrm{Mn} /$ planta, para plantas conduzidas com uma, duas e três hastes, respectivamente. Em estudo com espécies da família Asteraceae, Camargo et al. (2005) e Ferreira (2009) encontraram acúmulos de manganês da ordem de 2702,0 e $3388,0 \mu \mathrm{g} /$ planta em plantas de aster e crisântemo, respectivamente. Dechen \& Nachtigali (2006) relataram que, na condição de adequado suprimento de $\mathrm{Mn}$, as folhas acumulam altas concentrações conforme avançava a idade da planta, sendo uma pequena quantidade do elemento redistribuída das folhas velhas para as novas, em função da baixíssima mobilidade no floema.

As médias dos acúmulos de $\mathrm{Zn}$ nas folhas não apresentaram diferenças significativas para os diferentes sistemas de condução das plantas aos $0,15,30$, 45, 60 e 75 DAP, enquanto aos 90 DAP o sistema de condução com uma haste proporcionou acúmulo de zinco superior aos demais sistemas de condução (Tabela 3). O acúmulo de zinco, em plantas conduzidas com uma, duas e três hastes foi ajustado ao modelo de regressão cúbico (Figura 2). Nas plantas conduzidas com uma, duas e três hastes, o acúmulo 


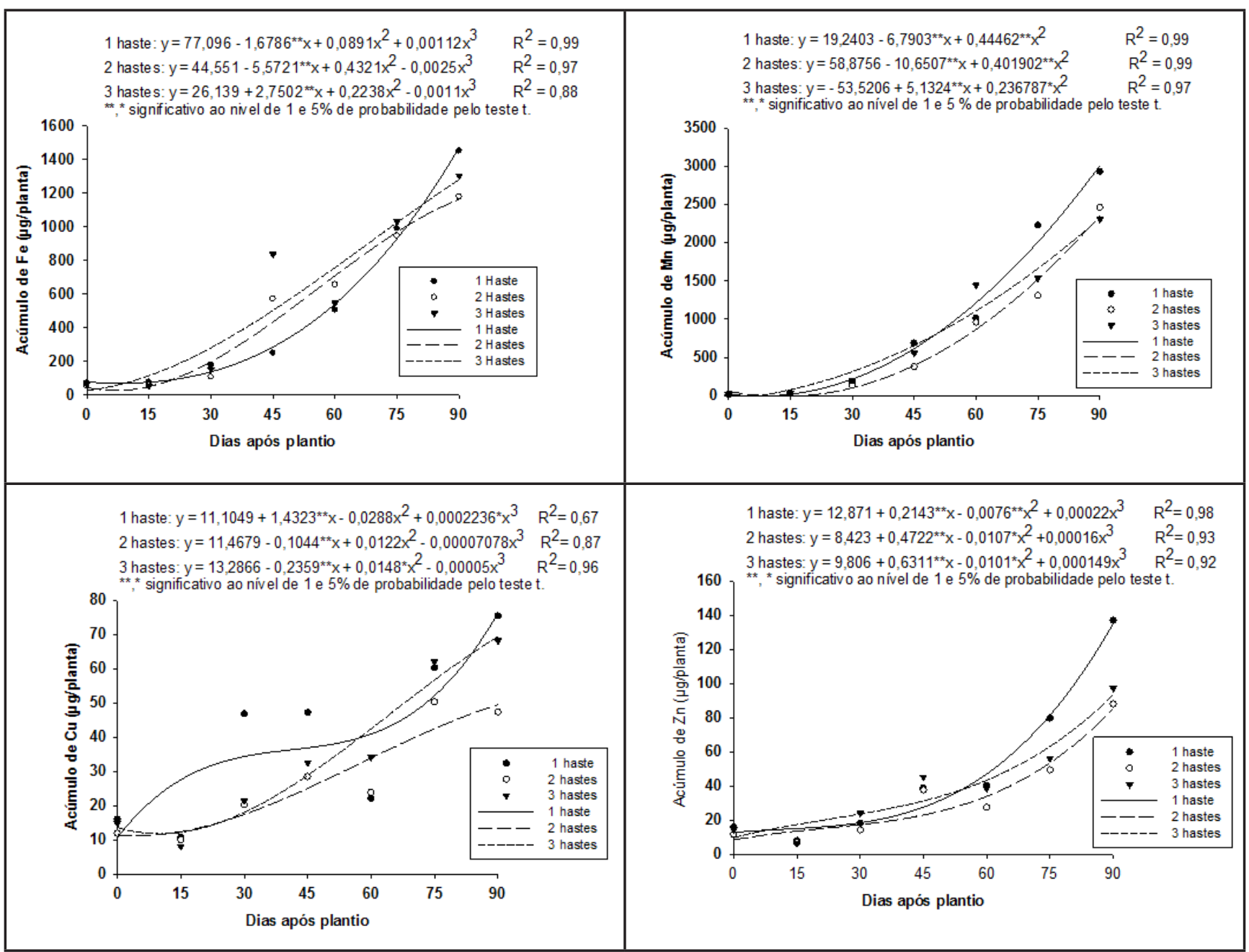

Figura 2. Acúmulo de ferro, cobre, manganês e zinco ( $\mu$ g/planta) em folhas de plantas de tango (y) conduzidas com uma, duas e três hastes em função dos dias após plantio (accumulation of iron, copper, manganese and zinc ( $\mu \mathrm{g} / \mathrm{plant}$ ) in leaves of goldenrod plants (y) conducted with one, two and three branches depending on days after planting). Viçosa, UFV, 2008.

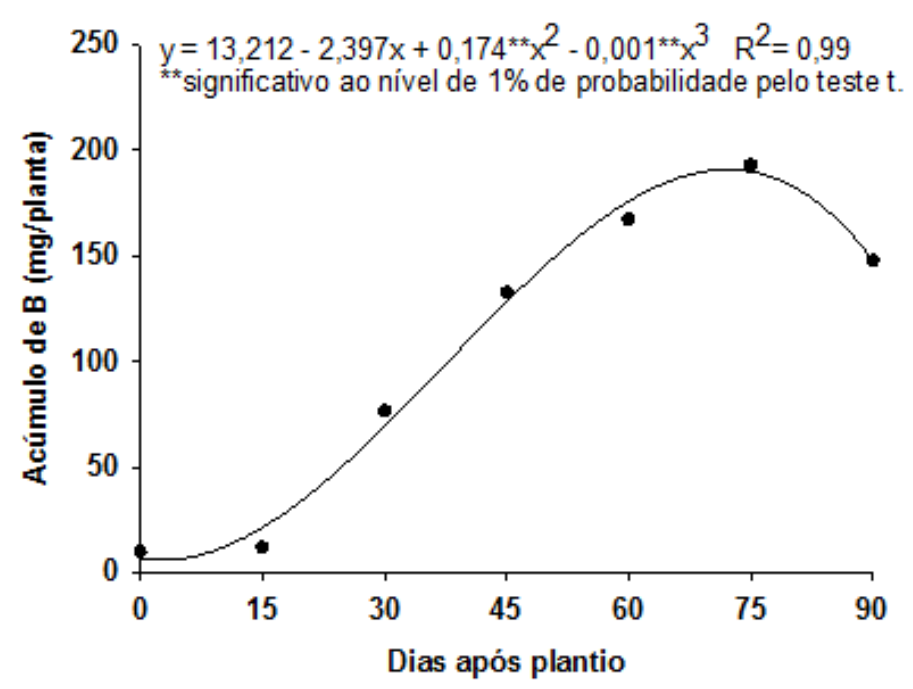

Figura 3. Acúmulo de boro ( $\mu \mathrm{g} / \mathrm{planta})$ em folhas de plantas de tango (y) conduzidas com uma, duas e três hastes em função dos dias após plantio (accumulation of boron ( $\mu \mathrm{g} / \mathrm{plant}$ ) in leaves of goldenrod plants (y) conducted with one, two and three branches depending on days after planting). Viçosa, UFV, 2008. máximo estimado ocorreu aos 90 dias após o plantio, sendo de 135,$34 ; 85,41$ e $93,57 \mu \mathrm{g} /$ planta, respectivamente. Camargo et al. (2005) observaram acúmulo de zinco em plantas de aster da ordem de $1025,0 \mu \mathrm{g} /$ planta aos 90 dias após plantio, valor em média 9,7 vezes maior que o observado em tango. Fernandes (2006) obteve 1052,9 e 6454,5 $\mu \mathrm{g} / \mathrm{planta}$ com crisântemo, cultivar Salmon Reagan, durante cultivo de inverno e verão.

As plantas conduzidas sob diferentes sistemas não mostraram diferença quanto ao acúmulo de boro nas folhas em todas as épocas avaliação (Tabela 3). Durante o ciclo de crescimento, o acúmulo de $\mathrm{B}$ acompanhou a produção da massa seca (Figura 3), observando-se que o máximo valor acumulado foi estimado em 190,9 $\mu \mathrm{g}$ de B/planta, aos 72 DAP. Esse valor foi inferior ao 
observado por Camargo et al. (2005) que encontraram acúmulo de 514,0 $\mu \mathrm{g}$ de B/planta em aster, aos 96 dias após plantio das mudas.

Pelos resultados obtidos pode-se concluir que o número de hastes por planta não influenciou significativamente o acúmulo de micronutrientes, sugerindo-se que as plantas sejam conduzidas com 3 hastes; o acúmulo de micronutrientes nas folhas acompanhou as curvas de ganho de massa seca de folha, tendo sido crescente durante todo o ciclo, obedecendo à ordem: $\mathrm{Mn}>\mathrm{Fe}>\mathrm{B}>\mathrm{Zn}>\mathrm{Cu}$.

\section{REFERÊNCIAS}

CAMARGO MS; MELLO SC; ANTI GR; CARMELLO QAC. 2005. Crescimento e absorção de nutrientes pelo Aster ericoides cultivado em solo sob estufa. Horticultura Brasileira 23: 271-274.
CUQUEL FL; KEIGO M; OTHON A. 1999 Poda apical para uniformizar a colheita de flores de 'tango'. Scientia agricola 56: 1009-1012.

DECHEN AR; NACHTIGALI CR. 2006. Micronutrientes. In: FERNADES MS (ed). Nutrição mineral de plantas. 1ed. SBCS. Viçosa. p.320-350

FERREIRA LDB. 2009 Características fitotécnicas e acúmulo de nutrientes em cultivares de crisântemo para vaso, em Goianira-GO. Goiânia: UFG. 132p. (Tese doutorado).

FERNANDES EP. 2006. Crescimento e marcha de absorção de nutrientes de crisântemo (Dendranthema grandiflorum, cv. Salmon Reagan) para corte, no período de inverno e verão. Goiânia: UFG. 121p. (Tese doutorado).

KIRKBYEA; RÖMHELDV. 2007. Micronutrients in plant physiology: functions, uptake and mobility, Proceedings 543, The International Fertiliser Society, P.O. Box 4, York, YO32 $5 Y S$, United Kingdom.

LORENZI H; SOUZA HM. 2001. Plantas ornamentais no Brasil: arbustivas, herbáceas e trepadeiras. $3^{\text {a }}$ ed.

MARSCHNER H. 1995. Mineral nutrition of higher plants. Londres: Academic. 889p.

MUNIZ MA; BARBOSA JG; GROSSI JAS;
ORBES MY; SÁ PG. 2009. Produção e qualidade de crisântemos de vaso fertirrigados com diferentes relações nitrato/amônio. Bioscience journal 25: 75-82.

PAULUS D; MEDEIROS SLP; SANTOS OS; MANFRON PA; PAULUS E; FABBRIN E. 2007. Teor e qualidade do óleo essencial de menta (Mentha arvensis L.) produzida sob cultivo hidropônico e em solo. Revista Brasileira de Plantas Medicinais 9: 80-87.

PEDROSA MW. 1998. Crescimento e acúmulo de nutrientes pela Gipsophila paniculata L. em cultivo hidropônico. Viçosa: UFV. 70p. (Dissertação mestrado).

TAIZ L; ZEIGER E. 2008. Fisiologia Vegetal. 4 ed. Porto Alegre: Artmed, 820p.

VEILING 2011 Critérios de padrão e qualidade Disponível em: http://www.veiling.com.br/ qualidade.swf?fileName=Tango\%20Corte.swf. Acessado em: 02/05/2011.

WACHOWICZ CM; CARVALHO RIN. 2002. Fisiologia vegetal: produção e pós-colheita. Curitiba: Champagnat, $423 \mathrm{p}$. 